\title{
Betriebliche Weiterbildung für Ältere - eine Längsschnittanalyse mit den Daten des IAB- Betriebspanels
}
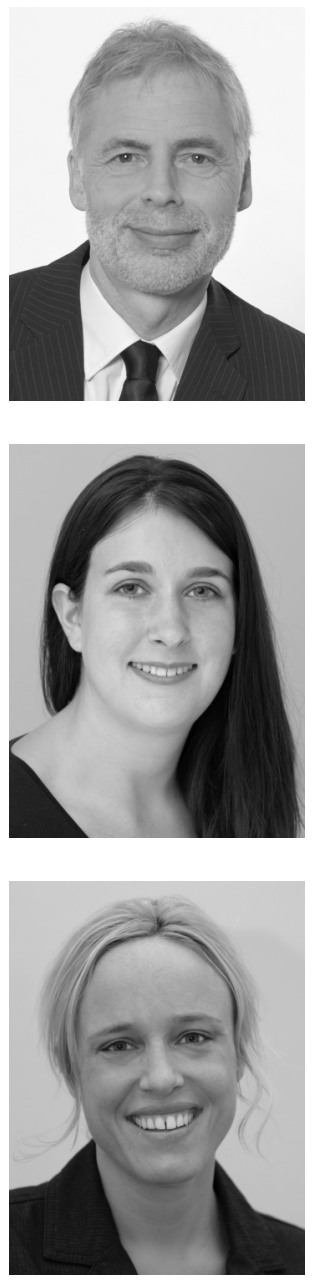

\section{Lutz Bellmann, Sandra Dummert, Ute Leber}

Betriebliche Weiterbildung, Ältere Arbeitnehmer, alternsbezogene Personalpolitik

Company-provided further training, older employees, age-related buman resource management

Bereits vorliegende empirische Ergebnisse zur Weiterbildung für Ältere weisen auf die geringe Verbreitung dieses Instruments in der betrieblichen Praxis hin. Während diese Studien auf einzelne Jahre bezogen sind, liegt dem vorliegenden Beitrag eine Längsschnittperspektive zugrunde. Auf Basis der Daten des IAB-Betriebspanels 2006-2011 wird für deutsche Betriebe gezeigt, dass ihr Weiterbildungsangebot Älteren gegenüber ansteigt, wenn der Untersuchungszeitraum ausgeweitet wird. Dennoch bleiben auch bei Betrachtung eines längeren Zeitraums die bekannten Erklärungsmuster der betrieblichen Weiterbildung für Ältere bestehen. So sind es insbesondere grössere Betriebe mit einer modernen technischen Ausstattung, einem hohen Anteil an qualifizierten Beschäftigten, einem hohen Anteil an älteren Beschäftigten und einer institutionalisierten Personalarbeit, die zumindest mit Unterbrechungen Weiterbildung für ihre älteren Mitarbeiter anbieten.

Previous empirical evidence shows that only a few firms offer further training for their older employees. While those studies focus on single years only, this paper has a longitudinal perspective. Using data from the IAB-Establishment Panel 2006-2011, it is shown that the share of German firms providing further training to their older employees increases when the observation period is enlarged. However, even in the long-term perspective we can still find almost the same factors explaining the provision of further training to older workers. Hence, the engagement in further training is higher in large, technically well-equipped firms which have high shares of qualified and older employees as well as an institutionalized human resource management.

\section{Einführung}

Die demografische Entwicklung wird in Deutschland - ebenso wie in den meisten anderen Industriestaaten - bereits in der nahen Zukunft zu einer Alterung und Schrumpfung des 
Erwerbspersonenpotenzials führen (zu entsprechenden Projektionen vgl. z.B. für die Europäische Union Population Europe 2012 und für Deutschland Fuchs/Söhnlein 2009). Während sich die geburtenstarken Jahrgänge schon in der Endphase ihrer Erwerbstätigkeit befinden oder kurz davor stehen, treten die geburtenschwachen Jahrgänge nach und nach in den Arbeitsmarkt ein. Hinzu kommt, dass durch den Altersaufbau der Erwerbstätigen, das Auslaufen von Frühverrentungen und den Anstieg der Regelaltersgrenze in der gesetzlichen Rentenversicherung mehr ältere Personen dem Arbeitsmarkt zur Verfügung stehen werden.

Bereits in den letzten Jahren ist in Deutschland die Erwerbsquote der über 50-Jährigen angestiegen und es wird davon ausgegangen, dass sie weiter zunehmen wird. Diese Entwicklung hat eine Reihe von Implikationen für den Arbeitsmarkt, die Unternehmen sowie die betriebliche Personalpolitik. Voraussetzung dafür, dass mehr Personen länger arbeiten können, ist grundsätzlich, dass sie bis ins Alter beschäftigungs- und arbeitsfähig bleiben. Neben der Frage, inwieweit Unternehmen dazu bereit sind, ältere Mitarbeiter einzustellen bzw. zu beschäftigen, kommt es dabei entscheidend auch auf die Leistungsfähigkeit, die Motivation und die Kompetenz der älteren Erwerbstätigen an.

In der Literatur und der Praxis werden verschiedene personalpolitische Massnahmen diskutiert, die dazu beitragen, dass Arbeitnehmer auch im Alter produktiv am Prozess der betrieblichen Leistungserstellung teilhaben können (vgl. z.B. Illmarinen 2006, 2012, Apel/ Fertig 2009, Brussig 2007, Morschhäuser 2006 oder Frerichs 2005). Dabei handelt es sich um Massnahmen des Gesundheitsschutzes, der Arbeitszeit- und der Arbeitsplatzgestaltung. Eine besondere Bedeutung wird im Rahmen der alter(n)sspezifischen ${ }^{1}$ Personalmassnahmen aber auch der Weiterbildung beigemessen: Nur wenn die Fähig- und Fertigkeiten der (älteren) Mitarbeiter aktualisiert und auf den neuesten Stand gebracht werden, ist es ihnen möglich, mit den Entwicklungen im organisatorischen und technischen Bereich Schritt zu halten und aktiv einen Beitrag zur Produktivität zu leisten. Zudem wird aus der psychologischen Forschung berichtet, dass sich die geistige Leistung durch Training verbessern lässt (vgl. z.B. Lindenberger/Baltes 1995).

Vorliegende empirische Untersuchungen auf der Basis von Betriebs- bzw. Unternehmensdaten zeigen jedoch, dass die Weiterbildung Älterer ebenso wie andere alter(n)sspezifische Personalinstrumente in der betrieblichen Praxis nur wenig verbreitet sind (vgl. z.B. Bellmann/Leber 2008 oder Brussig 2007 zu Ergebnissen aus dem IAB-Betriebspanel, Bannwitz 2008 zu Ergebnissen zur Weiterbildung aus der dritten Europäischen Erhebung über die berufliche Weiterbildung in Unternehmen (CVTS3) sowie Institut der deutschen Wirtschaft (IW) 2010 zu Befunden einer aktuellen Unternehmensbefragung des IW). So setzt nur ein geringer Anteil der Betriebe mit älteren Mitarbeitern derartige Massnahmen ein, und insbesondere die kleinen und mittleren Betriebe werden oftmals als „abstinent“ in der Weiterbildung Älterer, aber auch der Weiterbildung insgesamt, bezeichnet.

Die vorliegenden Studien betrachten die Verbreitung altersspezifischer Personalmassnahmen wie der Weiterbildung nur zu einem bestimmten Zeitpunkt bzw. innerhalb eines vergleichsweise kurzen Zeitraums. Daher stellt sich die Frage, wie sich das Angebot an Weiterbildung Älterer, das im Zentrum des vorliegenden Beitrags steht, darstellt, wenn der Untersuchungszeitraum ausgeweitet wird. Beteiligt sich die überwiegende Mehrzahl der

1 Der Begriff „altersspezifisch“ bezieht sich in der Regel auf Massnahmen, die im Alter ansetzen. Mit dem Begriff „alternsspezifisch“ werden hingegen Instrumente bezeichnet, die über den gesamten Erwerbsprozess hinweg Anwendung finden. 
Betriebe mit älteren Beschäftigten tatsächlich dauerhaft nicht an Weiterbildungsaktivitäten für die Angehörigen dieser Beschäftigtengruppe? Oder bieten Betriebe vielmehr Weiterbildung für ihre älteren Mitarbeiter nur unregelmässig an? Und welche Betriebe sind es, die zumindest manchmal, und welche, die nie aktiv in der Weiterbildung sind?

Fragen wie diese wurden in der empirischen Forschung bislang vernachlässigt. Neubäumer/Kohaut (2007) zeigen für die betriebliche Weiterbildung insgesamt, dass das Segment der weiterbildungsabstinenten Betriebe sinkt, wenn der Untersuchungszeitraum ausgeweitet wird. Wie sich aber das Weiterbildungsangebot für ältere Mitarbeiter in der längeren Perspektive darstellt, ist unbekannt. Daher verfolgt der vorliegende Beitrag das Anliegen, erstmals empirische Evidenz hierzu zu liefern. Auf Basis der Daten des IAB-Betriebspanels werden die Ergebnisse einer Längsschnittuntersuchung präsentiert, in der die „Kontinuität" von betrieblichen Weiterbildungsaktivitäten für Ältere analysiert wird. Zudem wird nach den Faktoren gefragt, die Einfluss darauf nehmen, ob bzw. wie regelmässig ein Betrieb in der längeren Perspektive Weiterbildung für seine älteren Mitarbeiter anbietet. Dies erscheint deswegen interessant, weil es denkbar ist, dass manche Betriebe nur im Rahmen konkreter Qualifizierungsanlässe wie z.B. der Einführung von Neuerungen weiterbilden, die nicht regelmässig, sondern nur in einzelnen Phasen anfallen. Darüber hinaus kann aber auch vermutet werden, dass es gewisse Rahmenbedingungen des betrieblichen Weiterbildungsverhaltens gibt - wozu etwa die Betriebsgrösse oder die Humankapitalausstattung gehören - die dazu führen, dass die Weiterbildung integraler (und damit möglicherweise auch regelmässiger) Bestandteil der Personalarbeit bzw. -entwicklung ist.

Der Beitrag ist wie folgt gegliedert: In Abschnitt 2 werden zunächst einige ausgewählte Befunde zur Weiterbildung Älterer skizziert. In Abschnitt 3 präsentieren wir die Ergebnisse unserer empirischen Analyse, bevor wir in Abschnitt 4 die zentralen Erkenntnisse zusammenfassen und Schlussfolgerungen ziehen.

\section{Weiterbildung für Ältere: Ausgewählte Ergebnisse}

Wie in der Einführung bereits erwähnt wurde, wird Weiterbildung als wichtig erachtet, um die Leistungsfähigkeit, die Motivation und die Kompetenz der Beschäftigten bis ins Alter hinein aufrecht zu erhalten. Auch wenn man sich darin einig ist, dass Weiterbildung (ebenso wie andere personalpolitische Massnahmen) über den gesamten Erwerbsprozess hinweg durchgeführt werden und nicht erst im Alter einsetzen sollte, spielt sie dennoch gerade auch für ältere Mitarbeiter eine wichtige Rolle. Insofern wird gefordert, dass Weiterbildung integraler Bestandteil einer alter(n)sspezifischen Personalpolitik sein sollte. Dabei wird insbesondere auch die Notwendigkeit einer regelmässigen, kontinuierlichen Weiterbildungsbeteiligung betont (vgl. z.B. Geldermann 2005, Frerichs 2005, Expertenkommission Finanzierung Lebenslangen Lernens 2004).

Die Weiterbildung Älterer ist Gegenstand einer Reihe von Untersuchungen. So beschäftigen sich verschiedene Disziplinen sowohl theoretisch als auch empirisch mit unterschiedlichen Aspekten der Qualifizierung von Personen in den oberen Altersgruppen. Im Folgenden sollen einige ausgewählte Ergebnisse aus diesen Studien dargestellt werden, wobei der Fokus insbesondere auf den vorliegenden empirischen Untersuchungen liegen soll.

Als erster Befund kann zunächst festgehalten werden, dass die Weiterbildungsbeteiligung älterer Personen unter der jüngerer liegt. So zeigen Individualdaten wie der Mikrozensus, das Sozio-oekonomische Panel oder der Adult Education Survey (früher: Berichtssystem Weiterbildung), dass die Teilnahme an Weiterbildung in den mittleren Altersgrup- 
pen zunächst ansteigt, dann aber (je nach Altersabgrenzung der verwendeten Studie) ab dem 50. bzw. 55. Lebensjahr wieder stark zurückgeht (vgl. z.B. Lois 2007, Leber/Möller 2008). Der Befund einer wesentlich geringeren Weiterbildungsinzidenz der Älteren wurde nicht nur für Deutschland sondern auch für insgesamt 25 andere Länder und auch für die Schweiz ermittelt (OECD 2012). Ebenso bestätigen auch Betriebsdaten die geringe Weiterbildungsbeteiligung Älterer: Nach den Ergebnissen der europäischen Weiterbildungserhebung CVTS weisen Beschäftigte ab 55 Jahren eine deutlich geringere Teilnahmequote an betrieblicher Weiterbildung auf als ihre jüngeren Kollegen. Dies gilt nicht nur für Deutschland, sondern auch für die anderen europäischen Staaten (vgl. Asplund 2005 oder Bannwitz 2008). Im IAB-Betriebspanel wird dagegen nicht nach der Teilnahmequote, sondern nach dem betrieblichen Angebot an Weiterbildung für Ältere (über 50-Jährige) gefragt. Danach gibt nur ein geringer Anteil der Betriebe, die überhaupt ältere Mitarbeiter beschäftigen, an, die Angehörigen dieser Gruppe auch in Weiterbildung einzubeziehen. Spezielle Weiterbildungsaktivitäten für Ältere, die z.B. etwaigen besonderen Lernbedürfnissen Rechnung tragen, werden dieser Datenquelle zufolge von noch weniger Betrieben praktiziert (vgl. Bellmann/Leber 2008). Das Angebot an betrieblicher Weiterbildung für ältere Mitarbeiter liegt dabei deutlich unter dem Weiterbildungsangebot für alle Beschäftigten und ist im Zeitverlauf relativ konstant geblieben. ${ }^{2}$ Für Grossbritannien wird darüber hinaus berichtet, dass ältere Arbeitnehmer häufiger als jüngere in vergleichsweise preiswerte On-thejob-Trainingsaktivitäten einbezogen werden bzw. an kürzeren Qualifizierungsmassnahmen teilnehmen (O'Mahony/Peng 2008).

Zur Erklärung der unterdurchschnittlichen Weiterbildungsbeteiligung Älterer werden in der Literatur sowohl das betriebliche Entscheidungskalkül als auch das der älteren Arbeitnehmer selbst herangezogen. Nimmt man zunächst die Perspektive der Individuen ein, so wird argumentiert, dass ältere Personen oftmals geringere Anreize haben, an Weiterbildung teilzunehmen. Warr (1993) bzw. Warr/Fay (2001) leiten ab, dass die Weiterbildungsmotivation mit dem Alter u.a. deswegen zurückgeht, weil die mit der Qualifizierungsbeteiligung verbundenen (finanziellen) Anreize bei älteren Beschäftigten geringer sind als bei jüngeren. Ähnlich weist auch Frerichs (2007) darauf hin, dass sich ältere Arbeitnehmer in der Vergangenheit oft nicht vor die Notwendigkeit gestellt sahen, an Weiterbildung teilzunehmen, weil es attraktive Pfade in einen vorzeitigen Ruhestand gab (vgl. hierzu Bellmann/Janik 2010), oder auch, weil es an entsprechenden Angeboten fehlte (Pannenberg 2007). Ein anderer Strang der Forschung argumentiert, dass sich ab einem gewissen Alter oftmals eine Zufriedenheit mit dem bisher Erreichten einstellt und dementsprechend die Notwendigkeit einer Weiterbildungsbeteiligung als nicht so bedeutsam eingeschätzt wird (vgl. z.B. Stamov-Roßnagel/Hertel 2010 oder Geldermann 2005).

Ältere Arbeitnehmer haben vorliegenden Untersuchungen zufolge nicht nur geringere Anreize sich weiterzubilden, sondern häufig auch gewisse Lernhemmungen. Solche können vor allem dann auftreten, wenn Personen bereits eine längere Zeit nicht mehr an Weiterbildungsmassnahmen teilgenommen oder in der Vergangenheit schlechte Erfahrungen mit dem Lernen gemacht haben. Von besonderer Bedeutung ist in diesem Zusammenhang auch die Unterscheidung zwischen kristalliner und fluider Intelligenz, die in der psychologischen Forschung getroffen wird. Während kristalline Intelligenz die Fähigkeit bezeich-

2 Nähere Angaben zu den konkreten Ergebnissen des IAB-Betriebspanels werden in Abschnitt 3.1 dargestellt. 
net, auf bereits Gelerntes zurückzugreifen, ist mit fluider Intelligenz die Fähigkeit beschrieben, Neues zu erlernen und für sich nutzbar zu machen. Es wird davon ausgegangen, dass die fluide Intelligenz im Alter abnimmt, und zwar vor allem dann, wenn längere Zeit nicht gelernt wird. Dies kann z.B. Konsequenzen für die Lerngeschwindigkeit haben und damit auch die Einstellung Älterer zum Lernen beeinflussen (vgl. z.B. Börsch-Supan et al. 2006 sowie Kanfer/Ackerman 2004).

Die Forschung weist allerdings auch darauf hin, dass es nicht ausreicht, allein auf das Merkmal „Alter“ abzustellen. Vielmehr gibt es innerhalb der Gruppe der Älteren zum Teil bedeutsame Unterschiede in der Weiterbildungsbeteiligung, die u.a. mit der Qualifikation der älteren Beschäftigten, dem Geschlecht oder anderer weiterbildungsrelevanter Faktoren zusammenhängen (vgl. Tippelt et al. 2009 oder Zwick 2011). So zeigen z.B. Analysen auf der Basis des Mikrozensus, dass höher qualifizierte ältere Arbeitnehmer häufiger an Weiterbildung teilnehmen als ihre geringer qualifizierten älteren Kollegen; das Gleiche gilt auch für ältere Beamte und Angestellte im Vergleich zu älteren Arbeitern (vgl. Leber/ Möller 2008 oder Lois 2007).

Richtet man das Augenmerk auf das betriebliche Entscheidungskalkül, so kann dieser Befund damit erklärt werden, dass Betriebe ihre Bildungsaktivitäten oftmals auf strategisch wichtige Kerngruppen ausrichten, zu denen die Älteren nicht zwangsläufig gehören. Dies geht auch einher mit vorliegenden Untersuchungen zu den betrieblichen Einflussbzw. Kontextfaktoren der Weiterbildung für Ältere (vgl. z.B. Bellmann et al. 2007 sowie Brussig 2007 zu den Determinanten altersspezifischer Personalpolitik insgesamt). Danach lässt sich das Angebot an Weiterbildung für Ältere einerseits als Folge besonderer, altersbezogener Bedingungen bzw. konkreter Qualifizierungsanlässe im Betrieb betrachten, andererseits aber auch im Kontext des generellen Human Resource Managements und damit der betrieblichen Strukturen bzw. Strategien (vgl. zu dieser Unterscheidung Brussig 2007). Im zuletzt genannten Kontext wurde z.B. gezeigt, dass Betriebe, in denen die über 50-Jährigen einen relativ hohen Anteil an der Belegschaft stellen, diese auch eher weiterbilden. Zum anderen investieren vor allem solche Betriebe in die Weiterbildung ihrer älteren Mitarbeiter, die ohnehin über ein ausdifferenziertes personalwirtschaftliches Instrumentarium verfügen. Dies trifft insbesondere auf Grossbetriebe zu, die deutlich häufiger Weiterbildung für Ältere anbieten als kleinere Betriebe. Dabei wird in diesen Betrieben zumeist nicht nur auf die Weiterbildung Älterer als ein Element der alter(n)sspezifischen Personalpolitik gesetzt, sondern vielmehr ein „Bündel“ entsprechender Massnahmen zum Erhalt der Beschäftigungsfähigkeit ergriffen.

Fragt man nach den konkreten Qualifizierungsanlässen, so wird insbesondere der Effekt von technischen und organisatorischen Neuerungen sowie von etwaigen Fachkräfteengpässen auf dem externen Arbeitsmarkt thematisiert. Für technische bzw. organisatorische Innovationen, wie die Neugestaltung von Beschaffungs- und Vertriebswegen, die Verlagerung von Verantwortung und Entscheidung nach unten, Einführung von Gruppenarbeit und die zunehmende Komplexität von Aufgaben, gilt generell, dass sie sich positiv auf die betriebliche Weiterbildung auswirken (vgl. z.B. Gerlach/Jirjahn 2001). In empirischen Studien, die speziell nach den Determinanten der betrieblichen Weiterbildung Älterer fragen, ist der Effekt hingegen nicht ganz so eindeutig (vgl. z.B. Bellmann/Leber 2011). Dies mag u.a. damit zusammenhängen, dass ältere Beschäftigte bei Neuerungen oftmals aussen vor bleiben und in besonderem Masse von Dequalifizierungsprozessen betroffen sind (vgl. Barkholdt et al. 1995; Beckmann 2007; Engelstädter/Sabu 2010 sowie Frerichs 2007). 
Demgegenüber zeigt sich im Hinblick auf Fachkräfteengpässe, dass Betriebe mit Rekrutierungsschwierigkeiten verstärkt ihre älteren Mitarbeiter weiterbilden. Die Qualifizierung Älterer bzw. damit einhergehend der Erhalt ihrer Beschäftigungsfähigkeit scheint somit eine Strategie zu sein, um einem etwaigen Fachkräftemangel zu begegnen (vgl. Bellmann/ Leber 2011).

Schliesslich gilt es aus betrieblicher Perspektive, auch die Auswirkungen der Weiterbildung, insbesondere auf den Unternehmenserfolg, zu berücksichtigen. Während es eine sehr umfangreiche Literatur zu den Produktivitätseffekten der Weiterbildung insgesamt gibt, liegen nur wenige Studien zu den Auswirkungen der Weiterbildung für Ältere vor. Göbel/ Zwick (2010) zeigen in einer Analyse verschiedener Personalmassnahmen, dass die Weiterbildung für Ältere nicht mit einer Erhöhung der Produktivität einhergeht. Dieses Ergebnis begründen sie damit, dass eine Weiterbildungsbeteiligung Älterer per se nicht mit positiven Effekten verbunden sein muss. Vielmehr komme es darauf an, in welcher Form die älteren Beschäftigten im Anschluss an die Weiterbildungsteilnahme eingesetzt werden. Ändert sich an ihrem Tätigkeitsfeld nichts, so bleibt auch ihre Produktivität auf dem gleichen Niveau.

\section{Empirische Analysen mit dem IAB-Betriebspanel}

Die vorangegangenen Ausführungen haben zweierlei deutlich gemacht: Zum einen ist die Weiterbildungsbeteiligung Älterer bzw. das Weiterbildungsangebot der Betriebe für diese Personengruppe vergleichsweise gering. Zum anderen bestehen strukturelle Unterschiede in der Partizipation verschiedener Personen- bzw. Betriebsgruppen, z.B. im Hinblick auf die Betriebsgrösse. Wie in der Einleitung angemerkt wurde, beziehen sich diese Ergebnisse auf einen bestimmten, relativ kurzen Zeitraum. Um Aufschluss über das Weiterbildungsverhalten der Betriebe in einer längerfristigen Perspektive zu bekommen, werden die eben präsentierten Befunde im Folgenden durch die Ergebnisse einer Längsschnittanalyse ergänzt. Dazu wird zunächst das Weiterbildungsverhalten auf der betriebsindividuellen Ebene im Zeitverlauf analysiert, bevor anschliessend nach den Einflussfaktoren der Häufigkeit der betrieblichen Weiterbildung für Ältere in einer längeren Periode gefragt wird.

Datengrundlage unserer empirischen Untersuchung ist das IAB-Betriebspanel (zu näheren Informationen zum IAB-Betriebspanel vgl. Fischer et al. 2009). Hierbei handelt es sich um eine seit 1993 in Westdeutschland und seit 1996 auch in Ostdeutschland jährlich durchgeführte Befragung von mittlerweile rund 16.000 Betrieben. Befragt werden Arbeitgeber aller Branchen und Grössenklassen im Rahmen von in der Regel persönlich-mündlichen Interviews durch TNS Infratest Sozialforschung. Im IAB-Betriebspanel werden Informationen zu verschiedenen Bereichen der betrieblichen Geschäfts- und Personalpolitik erhoben. Neben jährlich abgefragten Standardfragen enthält der Fragebogen auch wechselnde Schwerpunktthemen. Der betriebliche Umgang mit älteren Arbeitnehmern war Gegenstand der Erhebungen in den Jahren 2006, 2008 und 2011.

Im Rahmen dieses Themenkomplexes werden die Betriebe zuerst danach gefragt, ob sie überhaupt ältere Mitarbeiter beschäftigen. Als „älter“ wird ein Mitarbeiter im IAB-Betriebspanel dann bezeichnet, wenn er mindestens 50 Jahre alt ist. Den Betrieben, die über 50-jährige Beschäftigte haben, wird daran anschliessend eine Liste mit verschiedenen Massnahmen, die sich auf die Beschäftigung älterer Arbeitnehmer beziehen, vorgelegt. Dabei handelt es sich neben der Altersteilzeit, der besonderen Ausstattung der Arbeitsplätze, der Herabsetzung der Leistungsanforderungen und der altersgemischten Besetzung von Arbeitsgruppen auch um die Items „Einbeziehung Älterer in die betrieblichen Weiterbil- 
dungsaktivitäten“ und „Spezielle Weiterbildungsangebote für Ältere“. Auch wenn grundsätzlich angenommen werden kann, dass zwischen beiden Items gewisse Unterschiede bestehen (so kann z.B. vermutet werden, dass Betriebe vor allem dann spezielle Weiterbildung für Ältere tätigen, wenn sie von einem anderen Lernverhalten dieser Mitarbeitergruppe ausgehen, wohingegen sie bei einer generellen Einbeziehung Älterer in die Weiterbildung keine besondere Notwendigkeit erkennen, Ältere „anders“ weiterzubilden), werden in der folgenden Analyse beide Formen der Weiterbildung zusammengefasst. Ein Betrieb wird folglich dann als weiterbildungsaktiv bezeichnet, wenn er zumindest eine dieser Weiterbildungsarten praktiziert. Dies hat zum einen damit zu tun, dass insbesondere die spezielle Weiterbildung für Ältere nur sehr wenig verbreitet ist und die Fallzahlen daher keine tiefergehenden Analysen zulassen. Zum anderen ist es auch nicht das Erkenntnisinteresse unseres Beitrags, potenzielle Unterschiede oder Gemeinsamkeiten zwischen beiden Formen der Weiterbildung zu untersuchen.

\subsection{Betriebliche Weiterbildung für Ältere im Quer- und im Längsschnitt}

Betrachtet man zunächst die für uns relevante Betriebsbasis, d.h. die Betriebe mit älteren Beschäftigten, so zeigt sich den Daten des IAB-Betriebspanels zufolge, dass zur Mitte des Jahres $201173 \%$ aller Betriebe in Deutschland über 50-jährige Mitarbeiter beschäftigt haben (zu einer näheren Analyse der Frage, welche Betriebe ältere Mitarbeiter beschäftigen, vgl. Boockmann/Zwick 2004). Diese Durchschnittswerte werden stark durch das hohe Gewicht der Kleinbetriebe beeinflusst, die naturgemäss eine deutlich geringere Wahrscheinlichkeit aufweisen, zumindest einen älteren Beschäftigten zu haben. So lag der Anteil der Betriebe mit älteren Beschäftigten im Jahr 2011 im kleinbetrieblichen Segment (1 bis 19 Beschäftigte) bei $69 \%$, wohingegen bereits von den Betrieben mit 20 und mehr Mitarbeitern nahezu alle zum Stichtag zumindest einen älteren Mitarbeiter beschäftigt haben. Im Vergleich zu den Jahren 2006 und 2008 hat sich der Anteil der Betriebe mit älteren Arbeitnehmern relativ deutlich erhöht, wobei dieser Anstieg vor allem auf die Entwicklungen im kleinbetrieblichen Segment zurückzuführen ist (vgl. Tabelle 1).

\begin{tabular}{|l|c|c|c|}
\hline & 2006 & 2008 & 2011 \\
\hline 1 bis 19 Besch. & $56 \%$ & $59 \%$ & $69 \%$ \\
20 bis 249 Besch. & $96 \%$ & $96 \%$ & $96 \%$ \\
250 u.m. Besch. & $100 \%$ & $100 \%$ & $100 \%$ \\
insgesamt & $61 \%$ & $64 \%$ & $73 \%$ \\
\hline
\end{tabular}

Tabelle 1: Anteil der Betriebe mit über 50-jährigen Arbeitnehmern 2006, 2008 und 2011 (Quelle: IAB-Betriebspanel; querschnittsgewichtete Daten)

Wie im Rahmen des Literaturüberblicks in Abschnitt 2 erwähnt wurde, waren von den Betrieben mit über 50-jährigen Mitarbeitern nur wenige in deren Weiterbildung aktiv. Auch wenn die entsprechenden Befunde zur Verbreitung der Weiterbildung für Ältere bereits in anderen Beiträgen dargestellt sind (vgl. z.B. Bellmann/Leber 2008), sollen sie hier nochmals aufgegriffen werden, um so die Unterschiede zwischen der Quer- und der Längsschnittanalyse deutlich zu machen. Im Vergleich zu den bereits veröffentlichten Zahlen zur Weiterbildung für Ältere ist bei der folgenden Darstellung jedoch zu beachten, dass das zu- 
grunde liegende Sample ein anderes ist. So wurden in der Querschnittsbetrachtung (Tabelle 2) nicht alle Betriebe mit Angaben zu der Frage in der jeweiligen Welle berücksichtigt, sondern nur die, die in allen drei Jahren (2006, 2008 und 2011) beobachtet werden können. Dieses Vorgehen wurde gewählt, um die Ergebnisse von Quer- und Längsschnittanalyse besser vergleichen und somit interpretieren zu können. Grundsätzlich gilt jedoch, dass die hier dargestellten Befunde nur unwesentlich von den an anderer Stelle veröffentlichten abweichen, denen ein anderes Sample zugrunde liegt.

Insgesamt haben von den von uns berücksichtigten Betrieben mit über 50-jährigen Mitarbeitern $13 \%$ diese im Jahr 2011 weitergebildet, sei es durch die Einbeziehung Älterer in Qualifizierungsaktivitäten oder durch das spezielle Weiterbildungsangebot für Ältere (vgl. Tabelle 2). Dabei steigen die Weiterbildungsaktivitäten für Ältere mit der Betriebsgrösse an. Grössere Betriebe beziehen häufiger ihre älteren Mitarbeiter in die Weiterbildung ein bzw. bieten spezielle Qualifizierungsaktivitäten für diese an. Im Zeitverlauf ist festzustellen, dass das Weiterbildungsengagement der Betriebe Älteren gegenüber angestiegen ist. Haben im Jahr 2008 noch 8\% der Betriebe entsprechende Qualifizierungsaktivitäten angeboten, waren es im Jahr 2011 bereits 13\%. Ein Anstieg ist dabei in den Betrieben aller Grössenklassen festzustellen.

\begin{tabular}{|l|c|c|c|}
\hline & 2006 & 2008 & 2011 \\
\hline 1 bis 19 Besch. & $4 \%$ & $5 \%$ & $8 \%$ \\
20 bis 249 Besch. & $13 \%$ & $16 \%$ & $24 \%$ \\
250 u.m. Besch. & $39 \%$ & $45 \%$ & $54 \%$ \\
insgesamt & $7 \%$ & $8 \%$ & $13 \%$ \\
\hline
\end{tabular}

Tabelle 2: Betriebliche Weiterbildung für Ältere im Querschnitt (Basis: Betriebe mit älteren (über 50-jährigen) Beschäftigten und Beobachtungen für die Jahre 2006, 2008 und 2011; Quelle: IAB-Betriebspanel; querschnittsgewichtete Daten)

Diese Ergebnisse können den Schluss nahelegen, dass sich der Grossteil der Firmen mit älteren Beschäftigten nicht dauerhaft an der Weiterbildung dieser Mitarbeitergruppe beteiligt hat. Dies wäre dann der Fall, wenn es sich bei den in den einzelnen Jahren erfassten nicht-weiterbildenden Betrieben stets um dieselben Einheiten handeln würde. Um Aufschluss hierüber zu gewinnen, wird im Folgenden das Weiterbildungsverhalten auf der betriebsindividuellen Ebene im Zeitverlauf betrachtet. Dazu werden die Betriebe herangezogen, die in jedem Jahr ältere Beschäftigte haben. Diese werden in drei Gruppen eingeteilt:

- Betriebe, die regelmässig (d.h. in jedem der betrachteten Jahre) ihre älteren Mitarbeiter weitergebildet haben,

- Betriebe, die unregelmässig, d.h. nur in manchen der betrachteten Jahre, ihre älteren Mitarbeiter weitergebildet haben,

- Betriebe, die in keinem der betrachteten Jahre ihre älteren Mitarbeiter weitergebildet haben.

Als Untersuchungszeitraum werden die Jahre 2006, 2008 und 2011 herangezogen. Dabei werden nur die Betriebe berücksichtigt, die in jedem Jahr vollständige Angaben zur Frage 
nach der Beschäftigung Älterer sowie dem Engagement in der Weiterbildung gemacht haben.

Betrachtet man zunächst die Ergebnisse der entsprechenden Längsschnittanalyse, so zeigt sich, dass insgesamt 18\% der Betriebe mit älteren Beschäftigten die Angehörigen dieser Personengruppe mindestens einmal in den drei betrachteten Jahren weitergebildet haben. Dabei waren 2\% der Betriebe regelmässig in der Weiterbildung ihrer älteren Beschäftigten aktiv, wohingegen $15 \%$ in nur einem oder zwei Jahren ihre älteren Mitarbeiter weitergebildet haben. $82 \%$ der Betriebe mit Älteren haben in keinem der betrachteten Jahre ihre über 50-Jährigen weitergebildet (vgl. Tabelle 3).

\begin{tabular}{|l|c|c|c|c|}
\hline & \multicolumn{3}{|c|}{ Weiterbildung für Ältere in den Jahren 2006, 2008 und 2011 } \\
\hline & Mindestens einmal & $\begin{array}{c}\text { Davon: } \\
\text { regelmässig }\end{array}$ & $\begin{array}{c}\text { Davon: } \\
\text { manchmal }\end{array}$ & Nie \\
\hline 1 bis 19 Besch. & $10 \%$ & $*$ & $9 \%$ & $90 \%$ \\
20 bis 249 Besch. & $35 \%$ & $6 \%$ & $29 \%$ & $65 \%$ \\
250 u.m. Besch. & $72 \%$ & $22 \%$ & $50 \%$ & $28 \%$ \\
insgesamt & $18 \%$ & $2 \%$ & $15 \%$ & $82 \%$ \\
\hline
\end{tabular}

Tabelle 3: Betriebliche Weiterbildung für Ältere im Längsschnitt (2006/2008/2011) (Basis: Betriebe mit älteren (über 50-jährigen) Beschäftigten und Beobachtungen für die Jahre 2006, 2008 und 2011; Quelle: IAB-Betriebspanel; längsschnittgewichtete Daten, * Keine Angabe aufgrund zu geringer Fallzahl; Abweichungen in der Summe aufgrund von Rundungen möglich.)

Differenziert man die Ergebnisse weiter nach der Betriebsgrösse, so zeigt sich auch in der Längsschnittbetrachtung, dass der Anteil (zumindest unregelmässig) weiterbildender Betriebe mit der Betriebsgrösse ansteigt (vgl. Tabelle 3, sowie Tabelle 4 für einen verkürzten Untersuchungszeitraum (2008/2011)). In allen Grössenklassen liegt dieser Anteil über dem entsprechenden Anteil bei der rein stichtagsbezogenen Ermittlung (vgl. hierzu Tabelle 2). So waren es z.B. in den Jahren 2006, 2008 und 2011 immerhin gut sieben von zehn der grossen und gut drei von zehn der mittleren Betriebe, die ihre älteren Mitarbeiter mindestens einmal im Untersuchungszeitraum weitergebildet haben. Im Querschnitt 2011 waren dagegen „nur“ 54\% der grossen und 24\% der mittleren Betriebe weiterbildungsaktiv. Aber auch die kleinen Betriebe sind bei Ausdehnung des Betrachtungszeitraums tatsächlich weiterbildungsaktiver als es die Querschnittsergebnisse haben vermuten lassen: So haben sich in den Jahren 2006, 2008 und 2011 immerhin 10\% der Betriebe mit bis zu 19 Mitarbeitern (zumindest in manchen Jahren) an der Weiterbildung ihrer älteren Beschäftigten beteiligt, wohingegen es in den einzelnen Querschnitten jeweils nur 4\% (2006), 5\% (2008) bzw. 8\% (2011) waren. 


\begin{tabular}{|l|c|c|c|c|}
\hline & \multicolumn{4}{|c|}{ Weiterbildung für Ältere in den Jahren 2008 und 2011 } \\
\hline & $\begin{array}{c}\text { Mindestens } \\
\text { einmal }\end{array}$ & $\begin{array}{c}\text { Davon: } \\
\text { regelmässig }\end{array}$ & $\begin{array}{c}\text { Davon: } \\
\text { manchmal }\end{array}$ & Nie \\
\hline 1 bis 19 Besch. & $8 \%$ & $2 \%$ & $6 \%$ & $92 \%$ \\
20 bis 249 Besch. & $32 \%$ & $9 \%$ & $23 \%$ & $68 \%$ \\
250 u.m. Besch. & $69 \%$ & $38 \%$ & $30 \%$ & $31 \%$ \\
insgesamt & $16 \%$ & $4 \%$ & $11 \%$ & $84 \%$ \\
\hline
\end{tabular}

Tabelle 4: Betriebliche Weiterbildung für Ältere im Längsschnitt (2008/2011) (Basis: Betriebe mit älteren (über 50-jährigen) Beschäftigten und Beobachtungen für die Jahre 2008 und 2011; Quelle: IAB-Betriebspanel; längsschnittgewichtete Daten; Abweichungen in der Summe aufgrund von Rundungen möglich)

Zusammenfassend lässt sich somit festhalten: Bei Ausdehnung des Untersuchungszeitraums ist das Segment der nicht-weiterbildenden Betriebe zwar nach wie vor relativ hoch, doch ist es im Vergleich zu den Ergebnissen der Querschnittsbetrachtung gesunken. Die Befunde der Längsschnittanalyse legen somit nahe, dass der Anteil nicht-weiterbildender Betriebe tatsächlich kleiner ist als es aufgrund der reinen Querschnittsbetrachtung zu vermuten ist. Zusätzlich zu berücksichtigen ist, dass uns nicht für jedes Jahr aus dem IABBetriebspanel Informationen zur Weiterbildung für Ältere vorliegen. Es ist davon auszugehen, dass sich die Gruppe der nicht-weiterbildenden Betriebe nochmals verkleinern würde, wenn in unsere Analyse weitere Jahre einfliessen könnten.

\subsection{Betriebsstrukturelle Einflussfaktoren auf die betriebliche Weiterbildung für Ältere}

Weitet man den Betrachtungszeitraum also aus, so steigt der Anteil der (zumindest manchmal) weiterbildenden Betriebe. Wie die vorangegangenen Ausführungen gezeigt haben, gilt dies auch für die kleinen und mittleren Unternehmen, wenn auch zwischen den Betrieben der einzelnen Grössenklassen nach wie vor deutliche Unterschiede bestehen. Es ist davon auszugehen, dass sich die Gruppen der regelmässig, manchmal bzw. nie weiterbildenden Betriebe nicht nur im Hinblick auf die Betriebsgrösse, sondern auch bezüglich anderer Merkmale voneinander unterscheiden. So ist es denkbar, dass manche Betriebe nur im Rahmen konkreter Qualifizierungsanlässe wie z.B. der Einführung von Neuerungen weiterbilden, die nicht regelmässig, sondern nur in einzelnen Phasen anfallen. Darüber hinaus kann aber auch vermutet werden, dass es gewisse Rahmenbedingungen des betrieblichen Weiterbildungsverhaltens gibt - wozu etwa die Betriebsgrösse oder die Humankapitalausstattung gehören - die dazu führen, dass die Weiterbildung integraler (und damit möglicherweise auch regelmässiger) Bestandteil der Personalarbeit bzw. -entwicklung ist.

Um einen näheren Einblick in die betriebsstrukturellen Einflussfaktoren des betrieblichen Weiterbildungsengagements Älteren gegenüber zu gewinnen, werden im Folgenden die Ergebnisse einer Ordered-Probit-Analyse präsentiert, in der die drei Kategorien „regelmässig/wiederholt Weiterbildung für Ältere“, „manchmal Weiterbildung für Ältere“ und „(im Betrachtungszeitraum) keine Weiterbildung für Ältere“ die abhängigen Variablen darstellen. Dabei werden wieder die Jahre 2006, 2008 und 2011 als Untersuchungszeitraum verwendet. 
Für die in die Untersuchung einzubeziehenden betrieblichen Merkmale greifen wir auf die vorliegenden Studien zur Erklärung der betrieblichen Weiterbildung insgesamt bzw. der für ältere Mitarbeiter sowie solche zur altersspezifischen Personalpolitik zurück (zu den Determinanten der Weiterbildung insgesamt vgl. z.B. Gerlach/Jirjahn 2001, Düll/Bellmann 1998 oder Neubäumer/Kohaut 2007, zur Weiterbildung Älterer vgl. Bellmann et al. 2007 oder Bellmann/Leber 2008 sowie zur altersspezifischen Personalpolitik Brussig 2007). Diese Studien betten ihre empirische Analyse zumeist in einen humankapitaltheoretischen Rahmen (Becker 1964) ein, der jedoch um weitere theoretische Erklärungsansätze der New Training Literature (z.B. Acemoglu/Pischke 1999), den Segmentationsansatz (z.B. Sengenberger 1987) oder die Rational-Choice-Theorie (z.B. Esser 1990, 1991) ergänzt wird. Die betriebliche Entscheidung, Weiterbildung für Ältere anzubieten, wird danach grundsätzlich als Kosten-Nutzen-Kalkül verstanden, wonach Arbeitgeber dann dazu bereit sind, in die Qualifikationen ihrer älteren Mitarbeiter zu investieren, wenn der daraus (voraussichtlich) resultierende Ertrag die damit verbundenen Kosten übersteigt. Hinzu kommen Aspekte des Arbeitsmarktsegmentes, in dem die älteren Mitarbeiter beschäftigt sind, oder auch die generelle Einschätzung der Unternehmen älteren Beschäftigten gegenüber.

Unter Berücksichtigung dieser theoretischen Erklärungsansätze beziehen wir folgende Grössen in unsere empirische Analyse ein:

Merkmale der Personalstruktur: Zur Charakterisierung der Personalstruktur ziehen wir zum einen den Anteil der älteren Beschäftigten an der Belegschaft heran. Versteht man einen höheren Anteil der über 50-Jährigen im Betrieb als Indiz dafür, dass diese zur betrieblichen Kerngruppe gehören, kann dies die Wahrscheinlichkeit von Weiterbildungsinvestitionen (zumindest in manchen Perioden) erhöhen. Zum anderen berücksichtigen wir die Qualifikationsstruktur der Belegschaft, für die wir einen positiven Zusammenhang mit dem Weiterbildungsengagement erwarten. Begründet werden kann dies u.a. damit, dass sich qualifizierte Mitarbeiter bereits in der Vergangenheit als lernfähig erwiesen haben und hier besonders hohe Weiterbildungserträge zu erwarten sind.

Human Resource Management: Wie vorliegende empirische Studien (z.B. Käpplinger 2007) zeigen, hängt das Weiterbildungsverhalten von Betrieben insgesamt stark von der generellen Ausrichtung des Human Resource Managements ab. Von besonderer Bedeutung ist dabei der Grad der Institutionalisierung der Personalarbeit, was etwa damit erklärt werden kann, dass eine stärkere Erhebung und Planung z.B. des Qualifikationsbedarfs die Effektivität und damit die Erträge von Weiterbildungsaktivitäten erhöhen kann. Um die generelle personalpolitische Ausrichtung eines Betriebes zu charakterisieren, nehmen wir zunächst die Weiterbildungsintensität, d.h. die Weiterbildungsbeteiligung aller Beschäftigten, als erklärende Variable auf. Dabei vermuten wir, dass solche Betriebe eher ihre älteren Mitarbeiter in Qualifizierungsaktivitäten einbeziehen, die generell viel für die Weiterbildung tun. Zudem ziehen wir die Frage heran, ob es in einem Betrieb schriftlich fixierte Pläne für die Weiterbildung gibt. Schliesslich berücksichtigen wir auch den Einsatz weiterer altersbezogener Personalinstrumente im Betrieb, dabei konkret die Altersteilzeit, die altersgemischte Besetzung von Arbeitsgruppen und die besondere Ausstattung von Arbeitsplätzen. In welche Richtung der jeweilige Einfluss geht, dürfte dabei von der Art des Instruments abhängen: Während die Altersteilzeit (in der Ausprägung des Blockmodells) auf einen vorzeitigen Austritt aus dem Erwerbsleben abzielt und somit eher in einem negativen Zusammenhang mit der Weiterbildung stehen müsste, sind die beiden anderen Mass- 
nahmen auf den Erhalt bzw. Ausbau der Beschäftigungsfähigkeit älterer Mitarbeiter ausgerichtet und sollten komplementär zur Weiterbildung eingesetzt werden.

Technische Ausstattung: Eine moderne Betriebs- bzw. Kapitalausstattung geht in der Regel mit höheren Weiterbildungserträgen einher. Insofern gehen wir davon aus, dass Betriebe, die über eine moderne technische Ausstattung verfügen, eher in der Weiterbildung insgesamt, aber auch der ihrer älteren Mitarbeiter engagiert sind. Das gleiche dürfte auch für die Investitionstätigkeit eines Betriebes gelten, die u.U. einen konkreten Weiterbildungsbedarf induzieren kann. Speziell im Zusammenhang mit der Weiterbildung Älterer stellt sich jedoch die Frage, inwieweit diese Mitarbeiter in betriebliche Neuerungen einbezogen werden. Arbeiten sie eher mit älteren Produktionsverfahren, so kommt der für die Weiterbildung insgesamt bekannte Effekt hier möglicherweise nicht zum Tragen.

Kontext der Arbeitsbeziehungen: Manche Tarifverträge enthalten Regelungen zur Weiterbildung insgesamt, aber auch zu der von Älteren. Insofern gehen wir von einem positiven Einfluss der Tarifbindung auf die Weiterbildung für Ältere aus. Zudem ist in Betrieben mit Betriebsrat als institutionalisierte Arbeitnehmervertretung in Deutschland ein stärkerer (formalisierter) Einfluss der Belegschaft zu erwarten, der auch zu einer stärkeren Mitbestimmung in Personalfragen und hier speziell der Weiterbildung für Ältere, führen kann.

Betriebsgrösse und Branche: Schliesslich berücksichtigen wir die Betriebsgrösse und die Branchenzugehörigkeit. Grössere Betriebe verfügen über mehr Ressourcen und eine weiter ausgebaute Personalarbeit, was sich positiv auf die Weiterbildung für Ältere auswirken sollte. Hinzu kommt, dass hier die Fixkosten der Weiterbildung sowie die damit verbundenen Opportunitätskosten in der Regel geringer sind, während von vergleichsweise hohen und sicheren Weiterbildungserträgen ausgegangen werden kann. Zudem sind Unterschiede zwischen einzelnen Branchen zu erwarten, die etwa mit branchentypischen Gepflogenheiten oder Arbeitsbedingungen zu tun haben.

Auf welche Art und Weise die genannten Faktoren für die empirische Analyse operationalisiert wurden, ist in Tabelle 5 dargestellt.

Insgesamt haben wir drei verschiedene Modelle gerechnet, die sich darin unterscheiden, aus welchen Jahren die exogenen Variablen stammen. Zum einen haben wir die erklärenden Variablen mit Werten aus dem Jahr 2006 verwendet (also zu Beginn des Untersuchungszeitraums), zum anderen die aus den Jahren 2008 (Mitte des Untersuchungszeitraums) und 2011 (Ende des Untersuchungszeitraums). Wie im Folgenden ersichtlich werden wird, unterscheiden sich die Ergebnisse zwischen diesen drei Schätzungen nur unwesentlich voneinander. 


\begin{tabular}{|c|c|c|}
\hline Erklärende Variable & Operationalisierung & $\begin{array}{l}\text { Erwarteter } \\
\text { Einfluss }\end{array}$ \\
\hline \multicolumn{3}{|l|}{ Merkmale der Personalstruktur: } \\
\hline Anteil der älteren Mitarbeiter & $\begin{array}{l}\text { Anteil der über 50-Jährigen in fünf Ka- } \\
\text { tegorien }^{3}\end{array}$ & + \\
\hline Qualifiziertenanteil & $\begin{array}{l}\text { Anteil der Mitarbeiter mit abgeschlosse- } \\
\text { ner Lehre oder (Fach-) Hochschulab- } \\
\text { schluss zum 30.6. }\end{array}$ & + \\
\hline \multicolumn{3}{|l|}{ Human Resource Management: } \\
\hline Altersgemischte Arbeitsgruppen & Dummy $(1=\mathrm{ja} / 0=$ nein $)$ & + \\
\hline $\begin{array}{l}\text { Besondere Ausstattung der Arbeitsplätze } \\
\text { für Ältere }\end{array}$ & Dummy $(1=\mathrm{j} a / 0=$ nein $)$ & + \\
\hline Altersteilzeit & Dummy $(1=\mathrm{ja} / 0=$ nein $)$ & - \\
\hline Weiterbildungsbeteiligung insgesamt & $\begin{array}{l}\text { Anteil der Weiterbildungsteilnehmer an } \\
\text { den Beschäftigten im } 1 \text {. Halbjahr }{ }^{4}\end{array}$ & + \\
\hline $\begin{array}{l}\text { Schriftlich fixierte Pläne für die Personal- } \\
\text { entwicklung }\end{array}$ & Dummy $(1=\mathrm{ja} / 0=\text { nein })^{5}$ & + \\
\hline \multicolumn{3}{|l|}{ Technische Ausstattung: } \\
\hline Moderne technische Ausstattung & $\begin{array}{l}\text { Dummy }(1=j a / 0=\text { nein }) \text { für sehr moder- } \\
\text { ne/ moderne Ausstattung } 6\end{array}$ & + \\
\hline $\begin{array}{l}\text { Investitionen in Produktionsanlagen und/ } \\
\text { oder Informations- und Kommunikati- } \\
\text { onstechnologie }\end{array}$ & $\begin{array}{l}\text { Dummy }(1=j a / 0=\text { nein }) \text { für getätigte In- } \\
\text { vestitionen }\end{array}$ & + \\
\hline \multicolumn{3}{|l|}{ Kontext der Arbeitsbeziehungen: } \\
\hline Tarifbindung & Dummy $(1=\mathrm{j} a / 0=$ nein $)$ & + \\
\hline Betriebsrat & Dummy $(1=\mathrm{ja} / 0=$ nein $)$ & + \\
\hline \multicolumn{3}{|l|}{ Betriebsstruktur: } \\
\hline Betriebsgrösse & Logarithmierte Beschäftigtenzahl & + \\
\hline Branchenzugehörigkeit & 10 bzw. 11 Branchendummies $^{7}$ & \\
\hline
\end{tabular}

Tabelle 5: Operationalisierung der erklärenden Variablen und erwarteter Einfluss

3 Der Anteil der Älteren an der Belegschaft liegt gruppiert in fünf Ausprägungen vor: unter 10\% (1), 10 bis unter $20 \%$ (2), 20 bis unter $30 \%$ (3), 30 bis unter $50 \%$ (4), $50 \%$ und mehr (5).

4 Für die Weiterbildungsbeteiligung des Jahres 2006 wurden die Angaben der Betriebe aus der Welle 2005 herangezogen, da im IAB-Betriebspanel 2006 keine Daten zur Weiterbildung erhoben wurden.

5 Hierfür wurden ebenfalls für das Jahr 2006 die Angaben aus der Welle 2005 herangezogen.

6 Der Stand der technischen Ausstattung ergibt sich aus der Selbsteinschätzung der Betriebe auf einer Skala von 1 („neuester Stand“) bis 5 („völlig veraltet“). Für unsere Analyse wurden die Stufen 1 und 2 (im Vergleich zu 3 bis 5) zusammengefasst.

7 Aufgrund einer Umstellung der Klassifikation der Wirtschaftszweige aus dem Jahr 2003 (WZ03) auf die des Jahres 2008 (WZ08) im IAB-Betriebspanel im Jahr 2009 gingen in den Jahren 2006 und 2008 jeweils 10 Branchendummies und im Jahr 201111 Branchendummies in die Schätzungen ein. 
Betrachtet man die Ergebnisse der Orderd-Probit-Schätzungen (vgl. Tabelle 6), so ist zunächst festzustellen, dass das betriebliche Weiterbildungsengagement Älteren gegenüber wesentlich von der generellen personalwirtschaftlichen Ausrichtung des Betriebes beein-

\begin{tabular}{|c|c|c|c|}
\hline \multicolumn{4}{|c|}{ Abhängige Variable: „Regelmässigkeit“ der Weiterbildung } \\
\hline Exogene Variablen aus dem Jahr & 2006 & 2008 & 2011 \\
\hline \multicolumn{4}{|l|}{ Personalstruktur: } \\
\hline Anteil der über 50-jährigen Mitarbeiter & $\begin{array}{l}0,0615 * * * \\
(0,019)\end{array}$ & $\begin{array}{l}0,0882 * * * \\
(0,016)\end{array}$ & $\begin{array}{l}0,1155 * * * \\
\quad(0,017)\end{array}$ \\
\hline Qualifiziertenanteil & $\begin{array}{l}0,4622 * * * \\
(0,099)\end{array}$ & $\begin{array}{l}0,5655 * * * \\
(0,084)\end{array}$ & $\begin{array}{l}0,5224 * * * \\
(0,086)\end{array}$ \\
\hline \multicolumn{4}{|l|}{ Human Resource Management: } \\
\hline Altersgemischte Teams & $\begin{array}{l}0,9016 * * * \\
(0,056)\end{array}$ & $\begin{array}{l}0,9735 * * * \\
(0,050)\end{array}$ & $\begin{array}{l}0,8282 * * * \\
\quad(0,046)\end{array}$ \\
\hline $\begin{array}{l}\text { Besondere Ausstattung der Arbeitsplätze für } \\
\text { Ältere }\end{array}$ & $\begin{array}{l}0,2460 * * * \\
(0,088)\end{array}$ & $\begin{array}{l}0,1927 * * * \\
(0,072)\end{array}$ & $\begin{array}{l}0,3803 * * * \\
\quad(0,063)\end{array}$ \\
\hline Altersteilzeit & $\begin{array}{l}0,1955 * * * \\
(0,056)\end{array}$ & $\begin{array}{l}0,1211 * * \\
(0,051)\end{array}$ & $\begin{array}{l}0,0740 \\
(0,050)\end{array}$ \\
\hline Weiterbildungsbeteiligung insgesamt & $\begin{array}{l}0,4368 * * * \\
(0,075)\end{array}$ & $\begin{array}{l}0,5293 * * * \\
\quad(0,061)\end{array}$ & $\begin{array}{l}0,6009 * * * \\
\quad(0,060)\end{array}$ \\
\hline Weiterbildungspläne & $\begin{array}{c}0,2925 * * \\
(0,046)\end{array}$ & $\begin{array}{c}0,3235 * * \\
(0,042)\end{array}$ & $\begin{array}{l}0,2508 * * * \\
\quad(0,043)\end{array}$ \\
\hline \multicolumn{4}{|l|}{ Technische Ausstattung: } \\
\hline Moderne technische Ausstattung & $\begin{array}{c}0,0830 * \\
(0,049)\end{array}$ & $\begin{array}{c}0,1170 * * * \\
(0,042)\end{array}$ & $\begin{array}{c}0,1509 * * * \\
(0,041)\end{array}$ \\
\hline Investitionen & $\begin{array}{c}0,1912 * * * \\
(0,050)\end{array}$ & $\begin{array}{c}0,0955^{* *} \\
(0,042)\end{array}$ & $\begin{array}{l}0,0519 \\
(0,043) \\
\end{array}$ \\
\hline \multicolumn{4}{|l|}{ Kontext der Arbeitsbeziehungen: } \\
\hline Tarifbindung & $\begin{array}{r}-0,0032 \\
(0,051)\end{array}$ & $\begin{array}{l}0,0568 \\
(0,044)\end{array}$ & $\begin{array}{l}0,0018 \\
(0,045)\end{array}$ \\
\hline Betriebsrat & $\begin{array}{c}0,1604 * * * \\
(0,056)\end{array}$ & $\begin{array}{c}0,1443 * * * \\
(0,051)\end{array}$ & $\begin{array}{c}0,1173 * * \\
(0,052)\end{array}$ \\
\hline \multicolumn{4}{|l|}{ Betriebsstruktur: } \\
\hline Betriebsgrösse (log. Beschäftigtenzahl) & $\begin{array}{c}0,1787 * * * \\
(0,019)\end{array}$ & $\begin{array}{c}0,2122 * * * \\
(0,017)\end{array}$ & $\begin{array}{c}0,2146 * * * \\
\quad(0,017)\end{array}$ \\
\hline Branche (10 bzw. 11 Branchendummies) & ja & ja & ja \\
\hline Fallzahl & 3.549 & 5.358 & 5.308 \\
\hline Pseudo R ${ }^{2}$ & 0,1862 & 0,2372 & 0,2387 \\
\hline
\end{tabular}

Tabelle 6: Ergebnisse der Ordered-Probit-Schätzungen (Anmerkung: Signifikanzniveau: $* * *=\mathrm{p}<0,01 ;{ }^{* *}=\mathrm{p}<0,05 ;{ }^{*}=\mathrm{p}<0,1$; Standardfehler in Klammern; Quelle: IAB-Betriebspanel) 
flusst wird. So ist ein positiver Zusammenhang der Weiterbildung für Ältere mit der Weiterbildungsbeteiligung aller Beschäftigtengruppen auszumachen. Zudem erhöht eine institutionalisierte Personalarbeit (hier ausgedrückt durch das Vorhandensein von Weiterbildungsplänen) die Wahrscheinlichkeit, dass Betriebe ihre älteren Mitarbeiter zumindest manchmal weiterbilden. Auch der Einsatz weiterer altersspezifischer Personalinstrumente übt einen positiven Effekt auf das Engagement in der Weiterbildung für Ältere aus. Ein solcher lässt sich dabei nicht nur - wie erwartet - für die Instrumente „altersgemischte Arbeitsgruppen“ und „besondere Ausstattung der Arbeitsplätze“ feststellen, sondern überwiegend auch für die Altersteilzeit. Möglicherweise kann dieses Ergebnis damit erklärt werden, dass Informationen zur Weiterbildung sowie zur Altersteilzeit nur auf der Ebene des Betriebes, nicht aber der einzelner Mitarbeiter vorliegen. So gliedern Betriebe eventuell einzelne ältere Mitarbeiter aus, während sie bei anderen aktiv die Weiterbildung und damit die Beschäftigungsfähigkeit fördern. Denkbar ist z.B. aber auch, dass es einzelne Gruppen älterer Mitarbeiter im Unternehmen gibt, die durch die Teilnahme an Weiterbildung ihre Wettbewerbsposition jüngeren Kollegen gegenüber sichern bzw. verbessern wollen. Vorliegende empirische Ergebnisse zeigen, dass - zumindest in Ostdeutschland - mit der Weiterbildungsaktivität auch die Beschäftigungsstabilität der Älteren steigt (vgl. Bellmann et al. 2009).

Nicht nur die personalwirtschaftliche Ausrichtung des Betriebes, sondern auch die Personalstruktur beeinflusst sein Weiterbildungsengagement. Betriebe bilden dann ihre älteren Beschäftigten zumindest manchmal weiter, wenn sie über einen hohen Älteren- sowie einen hohen Qualifiziertenanteil verfügen. Zudem ergibt sich erwartungsgemäss ein positiver Effekt der Betriebsgrösse auf die Regelmässigkeit der Weiterbildung.

Nicht ganz eindeutig sind dagegen die Resultate im Hinblick auf die technische Ausstattung und den Kontext der Arbeitsbeziehungen: Zwar wirkt sich ein moderner technischer Stand positiv auf das Weiterbildungsverhalten eines Betriebes aus, doch lässt sich nicht in jedem Fall ein signifikanter Effekt für vom Betrieb getätigte Investitionen feststellen. Dies ist möglicherweise ein Indiz dafür, dass ältere Mitarbeiter in technische Neuerungen nicht immer eingebunden sind, so dass ein hierdurch ausgelöster Qualifizierungsbedarf nicht entsteht.

Während sich die Existenz eines Betriebsrates positiv auf die Regelmässigkeit der betrieblichen Weiterbildungsaktivitäten auswirkt, übt die Tarifbindung eines Betriebes keinen statistisch signifikanten Einfluss auf die Weiterbildung für Ältere aus.

Zusammenfassend lässt sich somit festhalten, dass ein grosser Einfluss betriebsstruktureller Merkmale auf das Weiterbildungsengagement Älteren gegenüber besteht. So sind es vor allem grössere Betriebe mit einem modernen technischen Stand, einem hohen Qualifiziertenanteil und einem hohen Anteil an älteren Beschäftigten, die zumindest manchmal ihre älteren Mitarbeiter weiterbilden. Zudem steht die Weiterbildung für die über 50-jährigen Beschäftigten aber auch in einem starken Zusammenhang mit der generellen personalwirtschaftlichen Ausrichtung eines Betriebes. Die Wahrscheinlichkeit, dass ältere Mitarbeiter in Qualifizierungsaktivitäten einbezogen werden, ist demnach dann vergleichsweise gross, wenn Betriebe über ein ausdifferenziertes personalwirtschaftliches Instrumentarium verfügen und ohnehin viel in die Weiterbildung ihrer Beschäftigten investieren.

Vergleicht man diese Befunde mit den Ergebnissen aus vorliegenden Querschnittsanalysen (vgl. insbesondere Bellmann et al. 2007 oder Bellmann/Leber 2008), so sind keine nennenswerten Unterschiede festzustellen. Auch bei Ausweitung des Betrachtungszeit- 
raums scheinen die bekannten Zusammenhänge zur Erklärung der Weiterbildung für Ältere weitgehend bestehen zu bleiben. Interessant erscheint vor allem der Effekt der Variablen, die wir zur Charakterisierung der Institutionalisierung der Personalarbeit herangezogen haben. Der sich hier ergebende positive Effekt ist bislang nur aus Studien zur Erklärung des betrieblichen Weiterbildungsverhaltens insgesamt bekannt; er scheint aber auch, wie hier gezeigt wurde, bei der Erklärung der Weiterbildung Älterer eine wichtige Rolle zu spielen.

\section{Fazit}

Im Fokus des vorliegenden Beitrags stand die betriebliche Weiterbildung für Ältere als ein zentrales Element der alter(n)sspezifischen Personalpolitik. Vor dem Hintergrund vorliegender empirischer Befunde zur geringen Verbreitung dieses Instruments wurden die Ergebnisse einer Längsschnittanalyse präsentiert, in der erstmalig die Bedeutung der Weiterbildung in einer längeren Perspektive betrachtet wurde. Dabei hat sich gezeigt, dass zwar auch bei Ausdehnung des Untersuchungszeitraums der Anteil nicht-weiterbildender Betriebe nach wie vor relativ hoch ist, dass er gegenüber der Betrachtung eines kürzeren Zeitraums aber doch abnimmt. So bietet ein gewisser Anteil der Betriebe mit älteren Arbeitnehmern zumindest unregelmässig, d.h. in manchen Jahren, Weiterbildung für diese Personengruppe an. Eine kontinuierliche Einbeziehung älterer Mitarbeiter in Weiterbildung, wie sie oftmals gefordert wird, ist jedoch nur bei einem sehr geringen Anteil der Betriebe zu finden.

Fragt man nach den Einflussfaktoren der „Häufigkeit“ von Weiterbildung für Ältere, so zeigt sich, dass insbesondere betriebsstrukturelle Merkmale wie die Betriebsgrösse und die Zusammensetzung der Belegschaft einen Effekt hierauf ausüben. Darüber hinaus steht die Weiterbildung für Ältere in einem engen Zusammenhang mit der generellen personalwirtschaftlichen Ausrichtung eines Betriebes. Eine weniger grosse Rolle bei der Erklärung des betrieblichen Weiterbildungsengagements Älteren gegenüber scheinen dagegen die Investitionstätigkeit sowie der Kontext der Arbeitsbeziehungen zu spielen. Insgesamt ist jedoch festzustellen, dass auch bei Ausdehnung des Betrachtungszeitraums im Grossen und Ganzen die gleichen Erklärungsmuster gelten wie in der kurzfristigen Perspektive.

Welche Schlussfolgerungen lassen sich aus diesen Befunden nun ableiten? Zunächst ist festzuhalten, dass es kaum ausreichend ist, die Weiterbildung Älterer nur in einem kurzen Zeitraum zu betrachten, will man Aussagen zur Bedeutung dieses Instruments in der betrieblichen Praxis treffen. Doch auch wenn ein längerer Untersuchungszeitraum zugrunde gelegt wird, ist der Anteil nicht-weiterbildender Betriebe nach wie vor als relativ hoch einzuschätzen. Angesichts der grossen Bedeutung, die diesem Personalinstrument zur Aufrechterhaltung der Leistungs- und Beschäftigungsfähigkeit beigemessen wird, scheinen hier verstärkte Anstrengungen zur Förderung wichtig. Dabei ist vor dem Hintergrund unserer Befunde vor allem an jene Betriebe zu denken, die auch über einen längeren Zeitraum hinweg weiterbildungsabstinent sind. Dies betrifft insbesondere kleine und mittlere Betriebe sowie solche, die über kein ausdifferenziertes Human Resource Management verfügen. Als möglicher Ansatzpunkt kann dabei etwa an entsprechende Beratungs- und Informationsangebote gedacht werden, denen auch als Instrument zur Steigerung der Weiterbildungsbeteiligung insgesamt eine wichtige Bedeutung beigemessen wird (vgl. z.B. Expertenkommission Finanzierung Lebenslanges Lernen 2004). 
Einschränkend zu unserer Analyse ist abschliessend anzumerken, dass auch hier aufgrund datentechnischer Restriktionen nur einzelne Jahre betrachtet werden konnten. Zudem ist davon auszugehen, dass die spezifische Fragestellung im IAB-Betriebspanel vor allem mit kursförmig organisierten Weiterbildungsformen in Verbindung gebracht wird. Insofern kann vermutet werden, dass das tatsächliche Ausmass der Weiterbildung für Ältere, das sich bei Einbeziehung weiterer Jahre und weiterer Lernarten ergeben würde, auch hier unterschätzt wird.

Interessant erscheint schliesslich auch eine vergleichbare Analyse auf der Basis anderer Datensätze, die neben Angaben zur Struktur der Betriebe auch nähere Informationen zur Struktur der (älteren) Weiterbildungsteilnehmer zur Verfügung stellen. Mit den Daten des Panels "Berufliche Weiterbildung als Bestandteil Lebenslangen Lernens" (WeLL) des Forschungsdatenzentrums im Institut für Arbeitsmarkt- und Berufsforschung (IAB) und der Erwerbstätigenbefragung des Bundesinstituts für Berufsbildung (BIBB) und der Bundesanstalt für Arbeitsschutz und Arbeitsmedizin (BAuA) könnten weitergehende Erkenntnisse gewonnen werden, da sich die Weiterbildungsbeteiligung älterer Beschäftigter in Abhängigkeit von individuellen Merkmalen wie dem Qualifikationsniveau oder der Stellung im Beruf unterscheidet.

\section{Literaturhinweise}

Acemoglu, D./Pischke, J. (1999): Beyond Becker: Training in imperfect labour markets, in: The Economic Journal, Jg. 109, Nr. 453, S. 112-142.

Apel, H./Fertig, M. (2009): Operationalisierung von „Beschäftigungsfähigkeit“- ein methodischer Beitrag zur Entwicklung eines Messkonzepts, in: Zeitschrift für ArbeitsmarktForschung, Jg. 42, Nr. 1, S. 5-28.

Asplund, R. (2005): The provision and effects of company training: a brief review of the literature, in: Nordic Journal of Political Economy, Jg. 31, Nr. 1, S. 47-73.

Bannwitz, J. (2008): Ältere Beschäftigte und betriebliche Weiterbildung. Ergebnisse des CVTS 3. Wissenschaftliches Diskussionspapier des BIBB, Nr. 104, Bonn.

Barkholdt, C./Frerichs, F./Naegele, G. (1995): Altersübergreifende Qualifizierung - eine Strategie zur betrieblichen Integration älterer Arbeitnehmer, in: Mitteilungen aus der Arbeitsmarkt- und Berufsforschung, Jg. 28, Nr. 3, S. 425-436.

Becker, G.S. (1964): Human Capital. A theoretical and empirical analysis, with special reference to Education, Chicago.

Beckmann, M. (2007): Age-biased technological and organizational change: firm-level evidence and management implications, WWZ-discussion paper 05/07, WWZ-Center of Business and Economics, University of Basel.

Bellmann, L./Janik, F. (2010): Betriebe und Frühverrentung: Angebote, die man nicht ablehnt, in: Zeitschrift für ArbeitsmarktForschung, Jg. 43, Nr. 4, S. 311-324.

Bellmann, L./Leber, U. (2011): Betriebliche Weiterbildung Älterer als Instrument zur Sicherung des Fachkräftebedarfs, in: Sozialer Fortschritt, Jg. 60, Nr. 8, S. 168-175.

Bellmann, L./Leber, U. (2008): Weiterbildung für Ältere in KMU, in: Sozialer Fortschritt, Jg. 57, Nr. 2, S. 43-48.

Bellmann, L./Leber, U./Stegmaier, J. (2007): Betriebliche Personalpolitik und Weiterbildungsengagement gegenüber älteren Beschäftigten, in: Loebe, H./ Severing, E. (Hrsg.): Demografischer Wandel und Weiterbildung. Strategien einer alterssensiblen Personalpolitik, Bielefeld, S. 81-97. 
Bellmann, L./Pahnke, A./Stegmaier, J. (2009): Betriebliche Weiterbildung und die Beschäftigung älterer Arbeitnehmer, in: Empirische Pädagogik, Jg. 23, Nr. 4, S. 431-459.

Boockmann, B./Zwick, T. (2004): Betriebliche Determinanten der Beschäftigung älterer Arbeitnehmer, in: Zeitschrift für ArbeitsmarktForschung, Jg. 37, Nr. 1, S. 53-63.

Börsch-Supan, A./Düzgün, I./Weiss M. (2006): Sinkende Produktivität alternder Belegschaften? Zum Stand der Forschung, in: Prager, J.U./Schleiter, A. (Hrsg.): Länger leben, arbeiten und sich engagieren. Chancen wertschaffender Beschäftigung bis ins Alter, Gütersloh, S. 85-102.

Brussig, M. (2007): Betriebliche Personalwirtschaft in einer alternden Erwerbsbevölkerung, in: Zeitschrift für Management, Jg. 2, Nr. 2, S. 198-223.

Düll, H./Bellmann, L. (1998): Betriebliche Weiterbildungsaktivitäten in West- und Ostdeutschland, in: Mitteilungen aus der Arbeitsmarkt- und Berufsforschung, Jg. 31, Nr. 2, S. 205-225.

Engelstädter, B./Sarbu, M. (2010): Enterprise software and service innovation: standardization versus customization, ZEW Discussion Paper No. 10-100.

Esser, H. (1991): Die Rationalität des Alltagshandelns. Eine Rekonstruktion der Handlungstheorie von Alfred Schütz, in: Zeitschrift für Soziologie, Jg. 20, Nr. 6, S. 430-445.

Esser, H. (1990): „Habits“, „Frames“ und „Rational Choice“, in: Zeitschrift für Soziologie, Jg. 19, Nr. 4, S. 231-247.

Expertenkommission Finanzierung Lebenslangen Lernens (2004): Finanzierung lebenslangen Lernens - der Weg in die Zukunft, Bielefeld.

Fischer, G./Janik, F./Müller, D./Schmucker, A. (2009): The IAB Establishment Panel. Things users should know, in: Schmollers Jahrbuch. Zeitschrift für Wirtschafts- und Sozialwissenschaften, Jg. 129, Nr. 1, S. 133-148.

Frerichs, F. (2005): Das Arbeitspotenzial älterer Mitarbeiterinnen und Mitarbeiter im Betrieb, in: Loebe, H./Severing, E. (Hrsg.): Wettbewerbsfähig mit alternden Belegschaften. Betriebliche Bildung und Beschäftigung im Zeichen des demografischen Wandels, Bielefeld, S. 49-58.

Frerichs, F. (2007): Altern als Lernhindernis? Bedingungen altersübergreifender Qualifizierung im demografischen Wandel, in: Sesselmeier, W./Lange, J./Bizer, K. (Hrsg.): Weiter Bildung! Berufliche Weiterbildung Älterer in kleinen und mittleren Unternehmen, Rehburg-Loccum, S. 207-236.

Fuchs, J./Söhnlein, D. (2009): Der Einfluss der Bevölkerungsentwicklung auf das künftige Erwerbspersonenpotenzial in Ost- und Westdeutschland, in: Cassens, I./Luy, M./Scholz, R. (Hrsg.): Die Bevölkerung in Ost- und Westdeutschland. Demografische, gesellschaftliche und wirtschaftliche Entwicklungen seit der Wende, Wiesbaden, S. 200-222.

Geldermann, B. (2005): Weiterbildung für die Älteren im Betrieb, in: Loebe, H./Severing, E. (Hrsg.): Wettbewerbsfähig mit alternden Belegschaften. Betriebliche Bildung und Beschäftigung im Zeichen des demografischen Wandels, Bielefeld, S. 69-79.

Gerlach, K./Jirjahn, U. (2001): Employer provided further training: Evidence from German establishment data, in: Schmollers Jahrbuch, Zeitschrift für Wirtschafts- und Sozialwissenschaften, Jg. 121, Nr. 2, S. 139-164.

Göbel, C./Zwick, T. (2010): Are personnel measures effective in increasing productivity of old workers? Labour Economics, Jg. 22, S. 80-93.

Illmarinen, J. (2006): Towards a longer worklife! Ageing and the quality of worklife in the European Union. Finnish Institute of Occupational Health, Helsinki, unter http://www.stm.fi/c/docu ment_library/get_file?folderId=39503\&name=DLFE-8602.pdf. 
Illmarinen, J. (2012): Promoting active ageing in the workplace. European Agency for Safety and Health at Work, unter: https://osha.europa.eu/en/publications/articles/promoting-active-ageing-i n-the-workplace.

Institut der deutschen Wirtschaft (IW) (2010): Ältere Beschäftigte: Meist hoch geschätzt, in: Informationsdienst des Instituts der deutschen Wirtschaft Köln (iwd), Jg. 36, Nr. 47, S. 3.

Kanfer, R./Ackerman, P. (2004): Aging, adult development and work motivation, in: Academy of Management Review, Jg. 29, Nr. 3, S. 440-458.

Käpplinger, B. (2007): Zusammenarbeit trotz Wettbewerb? Die Rollen von KMU und Weiterbildungsanbietern in Weiterbildungsnetzwerken, in: Sesselmeier, W./Lange, J./Bizer, K. (Hrsg.): Weiter Bildung! Berufliche Weiterbildung Älterer in kleinen und mittleren Unternehmen, Rehburg-Loccum, S. 143-160.

Leber, U./Möller, I. (2008): Weiterbildungsbeteiligung ausgewählter Personengruppen, in: Schmollers Jahrbuch, Zeitschrift für Wirtschafts- und Sozialwissenschaften, Jg. 128, Nr. 3, S. 405-429.

Lindenberger, U./Baltes, P.B. (1995): Testing-the-limits and experimental simulation: Two methods to explicate the role of learning in development, in: Human Development, Jg. 38, S. 349-360.

Lois, D. (2007): Determinanten der Weiterbildungsbeteiligung älterer Erwerbstätiger, in: Arbeit. Zeitschrift für Arbeitsforschung, Arbeitsgestaltung und Arbeitspolitik, Jg. 16, Nr. 1, S. 5-22.

Morschhäuser, M. (2006): Reife Leistung. Personal- und Qualifizierungspolitik für die künftige Altersstruktur, Berlin.

Neubäumer, R. (2007): Weniger Weiterbildung in kleinen und mittleren Unternehmen? Einordnung des Themas und erste empirische Ergebnisse, in: Sesselmeier, W./Lange, J./Bizer, K. (Hrsg.): Weiter Bildung! Berufliche Weiterbildung Älterer in kleinen und mittleren Unternehmen, RehburgLoccum, S. 87-95.

Neubäumer, R./Kohaut, S. (2007): A double hurdle approach for company further training behaviour and an empirical test of this using data from the IAB establishment panel, in: Zeitschrift für ArbeitsmarktForschung, Jg. 40, Nr. 2/3, S. 251-269.

OECD (2012): Education at a glance. Organization of Economic Cooperation and Development, Paris.

O’Mahony, M./Peng, F. (2008): Skill bias, age and organisational change. EU KLEMS Working Paper 36, Birmingham.

Pannenberg, M. (2007): Wer sät, der erntet? Individuelle Erträge von Weiterbildung in Deutschland, in: Sesselmeier, W./Lange, J./Bizer, K. (Hrsg.): Weiter Bildung! Berufliche Weiterbildung Älterer in kleinen und mittleren Unternehmen, Rehburg-Loccum, S. 97-108.

Population Europe (2012): Demographic Insights, No. 2: Active ageing \& solidarity between generations, unter: http://www.population-europe.eu/ext/document.ashx?id=0,3771,-1.

Sengenberger, W. (1987): Struktur und Funktionsweise von Arbeitsmärkten. Die Bundesrepublik Deutschland im internationalen Vergleich. Frankfurt/Main/New York.

Stamov-Roßnagel, C./Hertel, G. (2010): Older worker's motivation: against the myth of general decline, in: Management Decision, Jg. 48, Nr. 6, S. 894-906.

Tippelt, R./Schmidt, B./Schnurr, S./Sinner, S./Theisen, C. (2009): Bildung Älterer - Chancen im demographischen Wandel, Bertelsmann, Bielefeld.

Warr, P. (1993): In what circumstances does job performance vary with age?, European Work and Organizational Psychologist, Jg. 3, Nr. 3, S. 237-249. 
Warr, P./Fay, D. (2001): Short report: age and personal initiative at work, in: European Journal of Work and Organizational Psychology, Jg. 10, Nr. 3, S. 343-353.

Zwick, T. (2011): Why training older employees is less effective. ZEW Discussion Paper No 11-046, Mannheim.

Lutz Bellmann, Prof. Dr., ist Lehrstuhlinhaber für Volkswirtschaftslehre, insbes. Arbeitsökonomie, an der Friedrich-Alexander-Universität Erlangen-Nürnberg und Leiter des Forschungsbereichs Betriebe und Beschäftigung des Instituts für Arbeitsmarkt- und Berufsforschung Nürnberg.

Sandra Dummert, M.Sc., ist wissenschaftliche Mitarbeiterin im Forschungsbereich Betriebe und Beschäftigung des Instituts für Arbeitsmarkt- und Berufsforschung Nürnberg.

Ute Leber, Dr. rer. pol., ist wissenschaftliche Mitarbeiterin im Forschungsbereich Betriebe und Beschäftigung des Instituts für Arbeitsmarkt- und Berufsforschung Nürnberg.

Anschrift: Institut für Arbeitsmarkt- und Berufsforschung (IAB) der Bundesagentur für Arbeit (BA), Regensburger Str. 104, D-90478 Nürnberg 